\title{
Phlegmonous esophagitis with mediastinal abscess caused by pharyngeal abscess: a case description
}

\author{
Zaili Zhang, Meiru Jiang, Te Fang, Wenfei Tan \\ Department of Anesthesiology, the First Hospital of China Medical University, Shenyang, China \\ Correspondence to: Wenfei Tan. Department of Anesthesiology, the First Hospital of China Medical University, Nanjingbei Street 155\#, Shenyang, \\ China. Email: winfieldtan@hotmail.com.
}

Submitted May 30, 2021. Accepted for publication Sep 13, 2021.

doi: 10.21037/qims-21-573

View this article at: https://dx.doi.org/10.21037/qims-21-573

\section{Introduction}

Phlegmonous esophagitis is a rare event, especially when accompanied by a mediastinal abscess caused by a pharyngeal abscess. To date, there have been few reports in which these conditions have occurred simultaneously. In the case discussed in this report, the patient presented with all the above conditions. The patient underwent chest computed tomography (CT) to diagnose the cause of pharyngeal pain. He then received antibiotic treatment and underwent thoracic drainage in another hospital. However, one week later, he developed a fever, pharyngeal pain, and neck edema. He was diagnosed with phlegmonous esophagitis, pharyngeal abscess, and mediastinal abscess in our hospital. After antibiotic treatment, thoracic drainage, and a thoracotomy, the patient's vital signs were stable. However, he died on the first post-operative day. This report describes this case and considers the possible etiology.

Phlegmonous esophagitis characterized by esophageal stratification has rarely been reported, and its pathogenesis is unclear. In this report, we posit that a pharyngeal abscess caused both phlegmonous esophagitis and a mediastinal abscess-a potentially life-threatening infection most commonly associated with esophageal perforation or pharyngeal abscess (1). Each of these conditions could be life-threatening, and successful treatment requires multidisciplinary cooperation.

\section{Case presentation}

All procedures in studies involving human participants were carried out in accordance with the ethical standards of the institutional and/or national research committee(s) and with the Declaration of Helsinki (as revised in 2013). Written informed consent was obtained from the patient for publication of this case report and the accompanying images. A copy of the written consent is available for review by the editorial office of this journal.

In early January 2021, a 40-year-old male patient presented at the local hospital with a primary complaint of pharyngeal pain. None of the common causes of pharyngeal pain, such as toothache, trauma, or a foreign body, were observed. After a computed tomography (CT) scan of the chest, antibiotics were administered, and thoracic drainage was performed. However, the severity of the patient's pharyngeal pain progressively increased. We have not obtained further details of the initial treatment because it took place in a different hospital. One week later, the patient presented at the First Hospital of China Medical University with fever, pharyngeal pain, and neck edema. On January 13, 2021, a laryngeal CT showed a lamellar shadow in the right supraclavicular region (Figure 1A,B). The patient received rehydration with intravenous fluids and an anti-infective treatment (imipenem-cilastatin sodium). On January 16, 2021, contrast esophagography and chest CT showed esophageal stratification (Figure $1 C-1 F$ ).

The images suggested intramural dissection or external compression with possible erosion of the longitudinal muscle layer. The patient was diagnosed with phlegmonous esophagitis, a pharyngeal abscess, and a mediastinal abscess. On January 18, 2021, an emergency right thoracotomy, irrigation, curettage, and drainage were performed under 

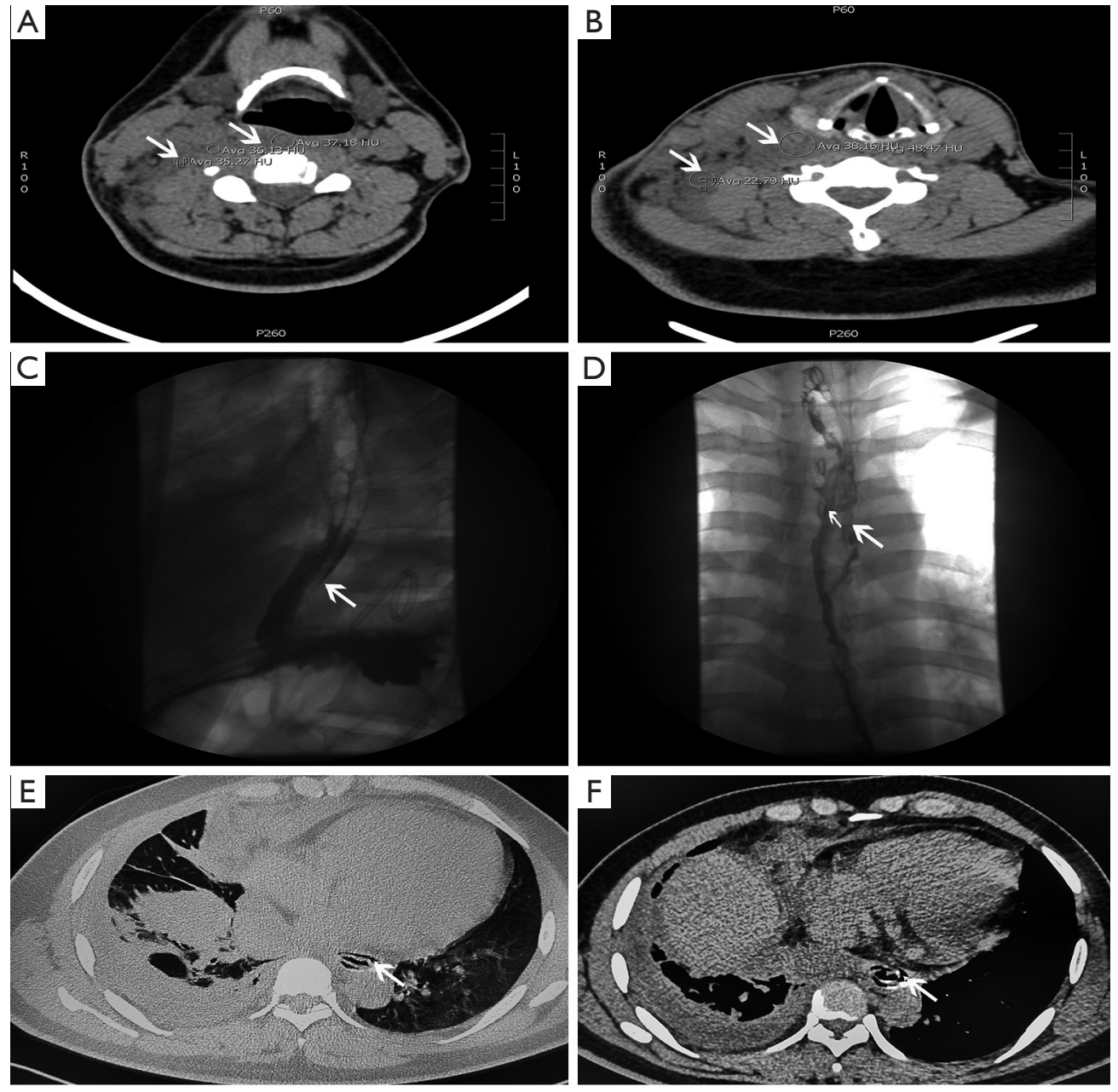

G

fig.a-b

fig.c-f

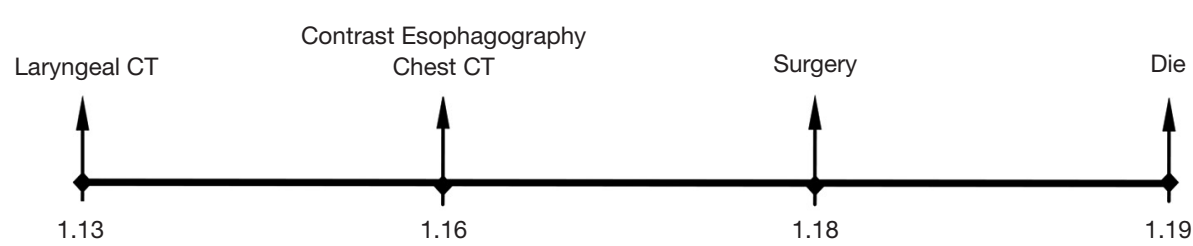

Figure 1 Typical images of the treatment process. (A,B) Laryngeal CT images showing a lamellar shadow (white arrow) in the right supraclavicular region. (C,D) Contrast esophagography showing that the contrast agent entered the area of esophageal stratification (white arrow). (E,F) Chest CT showing esophageal stratification (white arrow). (G) Summary of the entire process. CT, computed tomography.

general anesthesia. Large amounts of fibrous tissue and extensive adhesions were observed on the right side of the chest and subsequently dissected. Opening the mediastinal pleura, the thoracic surgeon observed a large amount of purulent fluid exudate and an edematous esophagus. The surgeon performed repeated flushing and inserted two drainage tubes. No significant abnormalities had been observed on the pre-surgery electrocardiogram (ECG), echocardiography, or laboratory tests. Post-surgery, the patient's vital signs were stable, and the drainage tubes were open. On January 19, 2021, the patient suddenly lost consciousness and went into cardiopulmonary arrest. $\mathrm{He}$ 
could not be resuscitated. The entire process is summarized in Figure $1 G$.

\section{Discussion}

Contrast esophagography and chest CT showed the formation of esophageal stratification, which has rarely been reported. The images suggest intramural dissection or external compression with possible erosion of the longitudinal muscle layer. Esophageal stratification is not a recognized medical condition of the esophagus or an established term in the field, and the observed phenomenon had not been identified in previous images. In this case, the esophageal stratification was defined as phlegmonous esophagitis, and the term was used to describe the unusual phenomenon seen in the images. There have only been two reported cases of phlegmonous esophagitis with mediastinitis caused by pharyngeal abscesses $(2,3)$. In the present case, we confirmed that pharyngeal abscesses could also cause phlegmonous esophagitis and mediastinitis. Pharyngeal abscesses extending to both the esophagus and mediastinum are often fatal, as is the rare phlegmonous esophagitis. We believe clinicians need to focus on obtaining images of phlegmonous esophagitis to assess patient risk. Further investigation is needed to determine the relationship between phlegmonous esophagitis and patient prognosis.

Esophageal perforation is a common cause of mediastinal abscesses and is associated with a high mortality rate (1). Pharyngeal abscesses have also been reported to result in mediastinal abscesses (4). The space posterior to the retropharyngeal space, which connects to the posterior mediastinum, is the primary route for the direct spread of infection (5). Descending necrotizing mediastinitis (DNM) is a life-threatening condition, especially when caused by a pharyngeal abscess. Early diagnosis, surgical drainage, and medical management in an intensive care unit may prevent patient mortality (6). In one review (7), three etiologies of mediastinitis were described: deep sternal wound infection related to sternotomy; esophageal perforation; and DNM secondary to oropharyngeal abscesses. In each case, the management strategy depended on the underlying etiology. Although these diseases are associated with a high risk of a poor outcome, a good prognosis is possible with aggressive treatment $(4,6)$.

In one report (8), a 34-year-old woman with pharyngeal and mediastinal abscesses developed septic shock and respiratory failure and died, despite aggressive treatment with broad-spectrum antibiotics, surgical drainage of the neck, and mediastinum, and cardiorespiratory support. The patient had gradually developed complications over two weeks, and the postmortem examination showed diffuse alveolar damage, bacterial thrombotic endocarditis, multiple thromboemboli in the pulmonary arteries, and ischemichypoxic injury to the brain. The patient in our case received similar treatment, including antibiotics, thoracic drainage, and thoracotomy, but went into sudden cardiopulmonary arrest the day after surgery, despite having displayed stable vital signs. Additionally, no significant abnormalities were observed on the pre-surgery ECG, echocardiography, and laboratory tests. Unfortunately, we did not perform a postmortem examination; however, we speculate that a severe inflammatory response led to hypercoagulability, which ultimately resulted in pulmonary infarction.

As recommended by Professor Yuichi Saito (3), physical examination, serum examination, chest radiography, computed tomography, bacterial culture of the mediastinal fluid, esophagogastroduodenoscopy (EGD), and esophagography were used to assess these diseases. In response to the disease processes, neck drainage, tracheostomy, antibiotic treatment, video-assisted thoracic surgery, and endoscopic procedures were effective.

\section{Conclusions}

In conclusion, despite aggressive treatment, phlegmonous esophagitis, mediastinal abscesses, and pharyngeal abscesses are associated with a high risk of mortality. Clinicians should be aware of the potential risks associated with phlegmonous esophagitis, especially when accompanied by a mediastinal abscess caused by a pharyngeal abscess.

\section{Acknowledgments}

Funding: None.

\section{Footnote}

Conflicts of Interest: All authors have completed the ICMJE uniform disclosure form (available at https://dx.doi. org/10.21037/qims-21-573). The authors have no conflicts of interest to declare.

Ethical Statement: The authors are accountable for all aspects of the work and ensuring that questions related to accuracy or integrity are appropriately investigated and resolved. 
All procedures in studies involving human participants were carried out in accordance with the ethical standards of the institutional and/or national research committee(s) and with the Declaration of Helsinki (as revised in 2013). Written informed consent was obtained from the patient for publication of this case report and the accompanying images. A copy of the written consent is available for review by the editorial office of this journal.

Open Access Statement: This is an Open Access article distributed in accordance with the Creative Commons Attribution-NonCommercial-NoDerivs 4.0 International License (CC BY-NC-ND 4.0), which permits the noncommercial replication and distribution of the article with the strict proviso that no changes or edits are made and the original work is properly cited (including links to both the formal publication through the relevant DOI and the license). See: https://creativecommons.org/licenses/by-nc-nd/4.0/.

\section{References}

1. Shiratori Y, Nakamura K, Ikeya T, Fukuda K. Treatment of esophageal perforation with mediastinal abscess by nasomediastinal drainage placement. Clin J Gastroenterol 2020;13:703-7.

2. Inaba Y, Tokano H, Ohtsu A, Kitamura K. A Case of

Cite this article as: Zhang Z, Jiang M, Fang T, Tan W. Phlegmonous esophagitis with mediastinal abscess caused by pharyngeal abscess: a case description. Quant Imaging Med Surg 2022;12(2):1632-1635. doi: 10.21037/qims-21-573
Descending Necrotizing MediastinitisPenetrating to the Esophagus. J Rural Med 2010;5:190-3.

3. Saito Y, Asami M, Miki A, Ehara Y, Abe K, Mochiki M, Yamauchi Y, Kodashima S, Sakao Y, Kawamura M. Deep Neck Infection Complicated by Phlegmonous Esophagitis and Mediastinitis. Ann Thorac Surg 2021;111:e403-6.

4. Kar A, Dharmic S, Suryanarayana V, Harish M. A case of mediastinitis secondary to retropharyngeal abscess. J Pharm Bioallied Sci 2015;7:S63-4.

5. Pulst-Korenberg A, Morris SC. Descending Necrotizing Mediastinitis Resulting from Pharyngitis with Perforation of the Aryepiglottic Fold. Case Rep Emerg Med 2020;2020:4963493.

6. Kilic D, Findikcioglu A, Ates U, Hekimoglu K, Hatipoglu A. Management of descending mediastinal infections with an unusual cause: a report of 3 cases. Ann Thorac Cardiovasc Surg 2010;16:198-202.

7. Pastene B, Cassir N, Tankel J, Einav S, Fournier PE, Thomas P, Leone M. Mediastinitis in the intensive care unit patient: a narrative review. Clin Microbiol Infect 2020;26:26-34.

8. Isaacs LM, Kotton B, Peralta MM Jr, Shekar R, Meden G, Brown LA, Raaf JH. Fatal mediastinal abscess from upper respiratory infection. Ear Nose Throat J 1993;72:620-2, 624-6, 631. 